\title{
МОДЕЛИРОВАНИЕ АКУСТИЧЕСКОГО КАРОТАЖА В ВЕРТИКАЛЬНО ТРАНСВЕРСАЛЬНО- ИЗОТРОПНЫХ СРЕДАХ С ПОГЛОЩЕНИЕМ
}

Лысь Е.B., Лисииа В.B.

(ИНГГ СО РАН, г.Новосибирск, Россия)

В работе представлен алгоритм расчёта волновых полей при акустическом каротаже, в вертикально трансверсально-изотрогньгх средах (VTI), учитывающй добротность горных пород. Алторитм базируется на применении линейной теории вязкоутругости. Построена конечно-разностная аппроксимация осесимметричной динамической задачи вязкоупругости, основанная на применении схемы на сдвинутых сетках(Верьё,1986), исследована устойчивость, проведен дисперсионный анализ. Для моделирования в неограниченных областях используются два подхода: идеально согласованный слой (англ. аббревиатура PML-perfectly matched layer) и продолокение целевой области на оптимальных сеткаx ( V.Druskin at all.).

При решении задач акустического каротажа, исклюочительно важно уметь эффективно рассчитывать волновое поле в некоторой известной модели. Далеко не все геологические объекты адекватно описываются моделью изотропной идеально упругой среды, не учитывающей такие важные факты, как диссипация энергин в реальньх сейсмических средах и анизотропия скоростей сейсмических волн.

Основополагающее соотношение для линейной теория вязкоупругости было получено Р.Кристенсоном (1982)

$$
\sigma_{i j}=G_{i j k t} * \dot{\varepsilon}_{k l}
$$

Здесь, * - подразумевает оператор свертки, точка над символом означает производную по времени. $G_{i j k l}$-тензор четвёртого ранга, содержащий функцию релаксация. Вместе с уравнение равновесия $\rho \dot{v}_{i}=\partial_{j} \sigma_{i j}$, мы получаем замкнутую систему, описывающую распространение волн в вязкоупругой среде.

Для того чтобы учесть поглощение энергии, мы должны определить механизм затухания. В работе [2] было показано, что «обобщённая стандартная линейная модель твердого тела»(англ.аббревиатура GSLS-generalized standard linear solid) даёт реалистичное описание волновых процессов, проходящих в земной коре, что подтверждают и экспериментальные данные. В рамках GSLS , мы получаем следущие выражения для функции релаксации:

$$
\left.G(t)=M_{R}\left(1+\sum_{l=1}^{L}\left(\frac{\tau_{\varepsilon l}}{\tau_{\sigma l}}-1\right)-t\right)^{-t / \tau_{\sigma l}}\right) \mathrm{H}(t)
$$

Здесь $M_{R}$-упругая постоянная, $\tau_{\varepsilon l}, \tau_{\sigma l}$ времена релаксации, $H(t)$-функция Хевисайда, Lчисло механизмов затухания.

Мерой диссипации энергик, в вязкоупругих средах служит параметр Q - добротность. Он определён, как число длин волн, после прохождения, которых первоначальный импульс затухнет в $e^{\pi}$ раз. В частотной области $Q=\frac{\operatorname{Re} G(\omega)}{\operatorname{Im} G(\omega)}$ поэтому тензор $Q_{i j}$ будет повторять структуру тензора упругих модулей в VTI и для определения заданной добротности возможно использование параметров аналогичных параметрам Томсона для скоростей упругих волн. Задавая несколько механизмов затухания, мы добиваемся частотной независимости в отределении добротности.

В докладе описан способ моделирования добротности называемый тау-методом, справедливый для значений $Q>10$ и позволяющий получить существенную экономию машинных ресурсов.

Известно, что для некоторых моделей анизотропии идеаљьо согласованный слой неустойчив, в этом случае предлагается распихять расчётную область с помошью оптимальных сеток, это позволяет избежать отражений от границ целевой области за расчётное время, при этом существенно экономятся вычислительные ресурсы по сравнению с расширением на равномерной сетке.

\section{ЛИТЕРАТУРА}

[1] Кристенсен Р. 1974. Введение в теорию вязкоупругости. Москва «Мир"

[2] Liu, H.P., Anderson, D.L., Kanamori, H., 1976. Velocity dispersion due to anelasticity: implications for seismology and mantle composition. Geophysical Journal of the Royal Astronomical Society 47, $41-58$.

[3] S.Asvadurov, V.Druskin, S.Moskow, Optimal grids for anisotropic problems. Wave propagation, ETNA , 2007, V.26;v.Lisitsa, Optimal grids for numerical solution of wave equation with variable coefficients, SJCM, 2005, v. 8(3)

[4] Joakim 0. Blanch, Johan 0. A. Robertsson and William W. Symes Modeling of a constant $\mathrm{Q}$ : Methodology and algorithm for an efficient and optimally inexpensive viscoelastic technique 